\title{
Nanowire nanoelectronics: Building interfaces with tissue and cells at the natural scale of biology
}

\section{Citation}

Cohen-Karni, Tzahi, and Charles M. Lieber. 2013. "Nanowire Nanoelectronics: Building Interfaces with Tissue and Cells at the Natural Scale of Biology." Pure and Applied Chemistry 85 (5) (January 11). doi:10.1351/pac-con-12-10-19.

\section{Published Version}

10.1351/pac-con-12-10-19

\section{Permanent link}

http://nrs.harvard.edu/urn-3:HUL.InstRepos:33464193

\section{Terms of Use}

This article was downloaded from Harvard University's DASH repository, and is made available under the terms and conditions applicable to Other Posted Material, as set forth at http:// nrs.harvard.edu/urn-3:HUL.InstRepos:dash.current.terms-of-use\#LAA

\section{Share Your Story}

The Harvard community has made this article openly available.

Please share how this access benefits you. Submit a story.

Accessibility 
Pure Appl. Chem., Vol. 85, No. 5, pp. 883-901, 2013.

http://dx.doi.org/10.1351/PAC-CON-12-10-19

(C) 2013 IUPAC, Publication date (Web): 11 April 2013

\title{
Nanowire nanoelectronics: Building interfaces with tissue and cells at the natural scale of biology*
}

\author{
Tzahi Cohen-Karni ${ }^{1,2, \ddagger}$ and Charles M. Lieber ${ }^{3}$ \\ ${ }^{1}$ David H. Koch Institute for Integrative Cancer Research, Cambridge, MA 02139, \\ USA; ${ }^{2}$ Boston Children's Hospital, Harvard Medical School, Boston, MA 02115, \\ USA; ${ }^{3}$ Department of Chemistry and Chemical Biology, School of Engineering and \\ Applied Sciences, Harvard University, Cambridge, MA 02138, USA
}

\begin{abstract}
The interface between nanoscale electronic devices and biological systems enables interactions at length scales natural to biology, and thus should maximize communication between these two diverse yet complementary systems. Moreover, nanostructures and nanostructured substrates show enhanced coupling to artificial membranes, cells, and tissue. Such nano-bio interfaces offer better sensitivity and spatial resolution as compared to conventional planar structures. In this work, we will report the electrical properties of silicon nanowires (SiNWs) interfaced with embryonic chicken hearts and cultured cardiomyocytes. We developed a scheme that allowed us to manipulate the nanoelectronic to tissue/cell interfaces while monitoring their electrical activity. In addition, by utilizing the bottom-up approach, we extended our work to the subcellular regime, and interfaced cells with the smallest reported device ever and thus exceeded the spatial and temporal resolution limits of other electrical recording techniques. The exceptional synthetic control and flexible assembly of nanowires (NWs) provides powerful tools for fundamental studies and applications in life science, and opens up the potential of merging active transistors with cells such that the distinction between nonliving and living systems is blurred.
\end{abstract}

Keywords: biotechnology; nano-bio interfaces; nanowires; synthesis.

\section{INTRODUCTION}

Recording electrical signals from cells and tissue is central to areas ranging from the fundamental biophysical studies of function in, for example, the heart and brain, through medical monitoring and intervention. Over the past several decades, studies of electroactive cells and tissue have been carried out by using a variety of recording techniques, including glass micropipette intracellular and patch-clamp electrodes [1,2], voltage-sensitive dyes [3,4], multielectrode arrays (MEAs) [5,6], and planar field-effect transistors (FETs) [7,8]. The latter two use well-developed microfabrication methods to allow for multiplexed detection on a scale not possible with micropipette technology, although the MEAs exhibit limited signal-to-noise $(\mathrm{S} / \mathrm{N})$ and relatively large detection areas that make cellular and subcellular recording immensely challenging $[9,10]$.

\footnotetext{
*Pure Appl. Chem. 85, 883-956 (2013). A collection of invited, peer-reviewed articles by the winners of the 2012 IUPAC Prize for Young Chemists.

¥Corresponding author
} 
A new class of molecular-scale electronic interfaces can be formed with cells and tissue using chemically synthesized semiconductor nanowires (NWs) as functional elements. These NWs have received intense interest in recent years, leading to the development of structures with rationally controlled geometry, composition, and electronic properties [11-13]. These characteristics have enabled semiconductor NWs to emerge as powerful building blocks for the bottom-up assembly of functional devices with applications areas from nanoelectronics [14-17] to the biosciences [18-21].

\section{The natural length scale of biology}

The span of length scale in biology varies by orders of magnitudes-from nanometer-sized nucleic or amino acids to a meters-scale whole organism. In between these two extreme limits, one can find protein molecules, such as ion channels, that are on the order of tens of nanometers, cells that are on the order of a few microns to hundreds of microns, and organs that are a few centimeters in length. Investigation of processes at the subcellular level necessitates spatial resolution at least in the order of the smallest building block of the cell-the molecular level, i.e., the nanoscale. These interfaces could be achieved through the use of nanostructures, such as NWs, with dimensions that are as small as a protein molecule.

\section{NWs as biological sensors}

Underlying biological detection using semiconductor NWs [18-21] is their configuration as FETs, which exhibit a conductance change in response to variations in the charge or potential at the surface of the channel region (Fig. 1A) [22]. This property makes FETs natural candidates for electrically based sensing since binding of a charged or polar biological or chemical species to the gate dielectric is analogous to the conventional case of applying a voltage gate using a metallic gate electrode (Fig. 1B). Significantly, NW FETs are more sensitive sensors than their planar counterparts because of their onedimensional (1D) nanoscale morphology. An analyte binding to the surface of an NW leads to depletion or accumulation of carriers in the "bulk" of the 1D nanometer-diameter structure, vs. only a shallow region near the surface in the case of a planar device. This unique feature of semiconductor NWs enables exquisite charge sensitivity that opens up new opportunities for interfaces with chemical and biological systems.
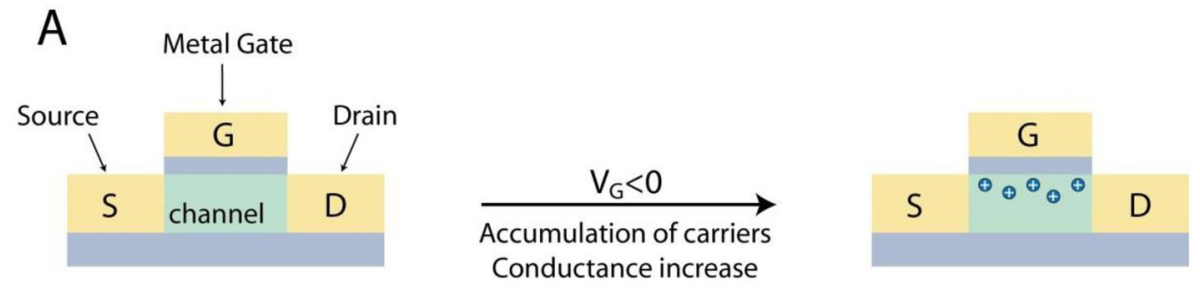

B
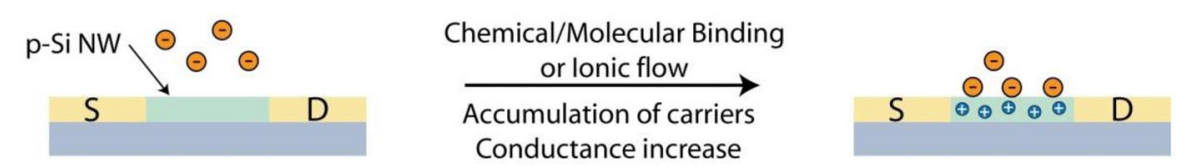

Fig. 1 Planar FET and NW FET (A) Schematic of a p-type planar FET device, where S, D, and G correspond to source, drain, and gate electrodes, respectively. (B) Schematic of electrically based sensing using a p-type NW FET, where binding of a charged biological or chemical species to the chemically modified gate dielectric is analogous to applying a voltage using a gate electrode. 
NWs elaborated as well-defined n- or p-channel FETs have been used as ultrasensitive sensors of chemical and biological species. In 2004, we achieved the limit of biological detection-single particle sensitivity - by detecting, in real time, the reversible and selective binding of virus particles to antibodymodified NW FETs [19]. The exquisite sensitivity of NW FETs was further demonstrated by sensing of a cancer marker protein, prostate specific antigen (PSA), down to approximately $75 \mathrm{fg} / \mathrm{ml}$, or approximately $2 \mathrm{fM}$ [20]; this represents a sensitivity limit 104-109 times below the state-of-the-art ion-sensitive planar FETs [23]. These sensitivities were also verified using top-down fabricated NWs [24]. In addition, using NW FET arrays we demonstrated multiplexed detection of multiple cancer markers with the same femtomolar sensitivity [20]. Recently, the use of SiNW FETs was extended to detection of DNA translocations. In this research we incorporated a SiNW short-channel sensor near a nanopore in a $\mathrm{SiN}_{x}$ thin membrane and used the highly sensitive FET to detect DNA translocations that are correlated with ionic current blockade events. These results will potentially provide a new class of nanopore sequencing devices for future investigations of DNA with high throughput and sensitivity [25].

\section{Advantages of nanoscale morphology for cellular interfaces}

A major advantage of NW FETs relates to the coupling between the NW and cells. The formation of a tight junction between a cell or cellular projections and the semiconductor surface is important in determining sensitivity, when measuring local field changes given the high ionic strength (ca. $150 \mathrm{mM}$ ) of cell culture medium. On a perfectly flat surface, the junction gap is on the order of tens of nanometers, a result of the finite size of adhesion/transmembrane proteins. NWs, however, are freestanding structures that protrude from the surface of the substrate and are expected to form naturally tighter junctions with the local cell membrane than is possible with a planar device (Figs. 2A and B).

Cellular adhesion, guidance, proliferation, and fate may be further enhanced by unique interactions between the nano-topographic surface and cell membrane [26-30]. For example, it has been demonstrated that nanostructured surfaces formed by carbon nanotube (CNT) networks or etched silica promote cellular adhesion, spreading, and guidance, even in the absence of conventional adhesion fac-

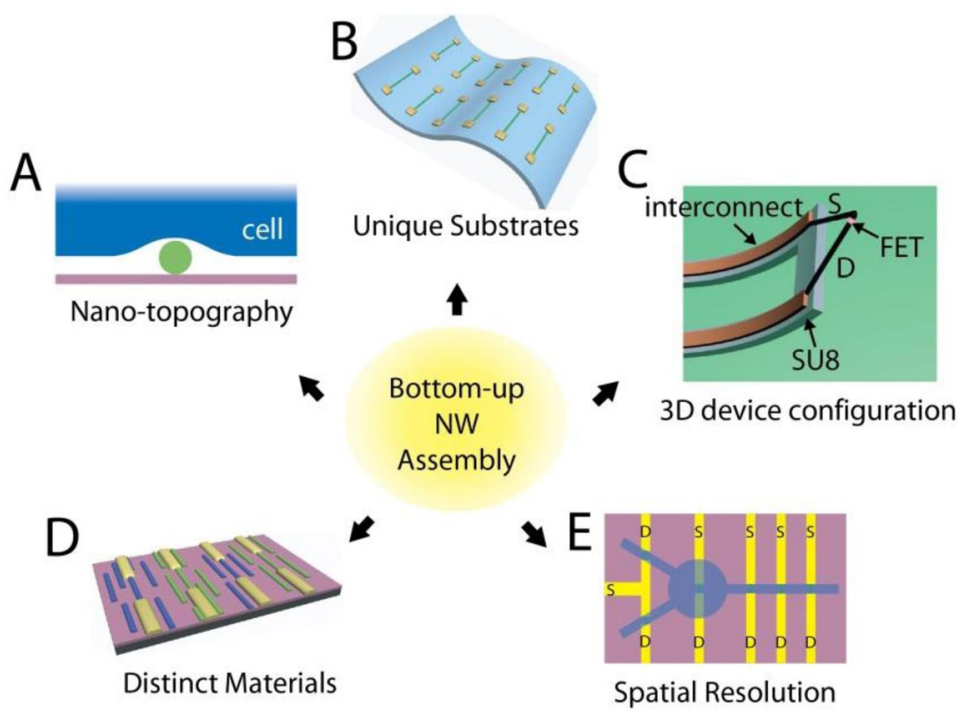

Fig. 2 Schematic diagram outlining unique advantages of bottom-up NW assembly. (A) Nanotopographic morphology, (B) ability to assemble devices on flexible, transparent substrates, (C) fabrication of unconventional 3D device configuration, (D) assembly of distinct NW materials on the same chip, and (E) high spatial resolution of NW devices. 
tors such as polylysine [31]. Moreover, sparse NW arrays were shown to direct axonal growth over large areas and with high spatial resolution; these arrays promoted the formation of focal adhesions, which are critical for tight cell-substrate junctions [32]. Taken together, these findings indicate that NW FETs could serve not only as sensitive electronic devices, but also as nanoscale interfaces that promote unique and favorable substrate-cell interactions.

One final aspect of nanostructured surfaces that warrants emphasis is their effect on ion-channel function. For example, results suggest that neurons cultured on CNT mats exhibit enhanced spiking activity (vs. those cultured on planar control surfaces), and thus that the conductive, nanostructured surface enhances membrane excitability [33,34]. While these studies warrant further mechanistic investigations, they provide additional evidence that nanostructured surfaces form unique interactions with the cell membranes not realized previously with planar electronic devices.

\section{FLEXIBLE NW DEVICE ARRAY INTERFACES WITH HEARTS}

Whole tissue, for example, the heart, represents a system where NW devices could be used to collect electrophysiological information. Activation sequences across the surface of the heart have been measured using macroscale metallic electrodes [35], optical microscopy of dyed tissue [3], and MEAs [6], but none of these techniques has been miniaturized to achieve single-cell resolution. Given the complexities of activation sequences, cellular- or subcellular-level interfaces with cardiac tissue could be crucial for better understanding cardiac dysfunction, such as, for example, arrhythmia [36].

\section{Characterization of heart/NW interface}

We used live hearts from embryonic chickens (E10-E15 stage) as a model system for studying tissue/NW interfaces. In a typical experiment with a planar NW FET chip configuration (Figs. 3A and B), a freshly isolated heart was placed on top of the active device region of a heated sample chamber [37]. The hearts beat spontaneously at a typical frequency of 1-3 Hz. Representative data of simultaneous recording from an NW FET and from a conventional glass pipette electrode inserted into the heart show close temporal correlation between initial sharp peaks, although the pipette peak occurs ca. $100 \mathrm{~ms}$ before the NW FET peak in each beat (Fig. 3C). The consistent time difference is expected since the pipette was inserted into a spatially remote region with respect to the NW FET devices. Examination of individual NW signals reveals an initial fast phase (full-width at half maximum, FWHM $=6.8 \pm 0.7 \mathrm{~ms}$ ) followed by a slower phase (FWHM $=31 \pm 9 \mathrm{~ms}$ ), where these two phases can be attributed to transient ion-channel current and mechanical motion, respectively. NW FET signals exhibiting the fast followed by slow phases were recorded in $85 \%$ of our $>75$ independent experiments, and thus demonstrate the reproducibility of our NW-based recording approach for tissue measurements [37].

The peaks recorded with our NW FETs (Fig. 3C) exhibit excellent S/N. The observed conductance changes associated with these peaks depend on the device sensitivity. To illustrate this point and provide voltage calibration for the peaks, data were recorded at a variety of applied water-gate potentials. NW FET results from a beating heart with the water-gate varied from -0.4 to $0.4 \mathrm{~V}$ show a decrease in the magnitude of the fast transient conductance change from ca. 55 to $11 \mathrm{nS}$, which is correlated with the decrease in device sensitivity over this same range of water-gate potentials [37]. Notably, the voltage-calibrated signal determined using the device sensitivity was essentially constant at $5.1 \pm 0.4 \mathrm{mV}$. These results confirm the stability of the interface between the NW FETs and beating heart, and highlight the necessity of recording explicit device sensitivity to interpret corresponding voltages [37]. 
A

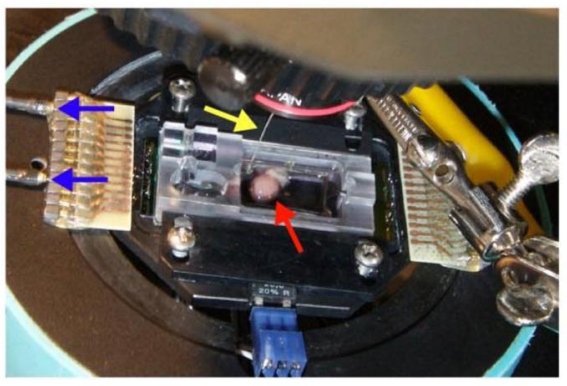

B

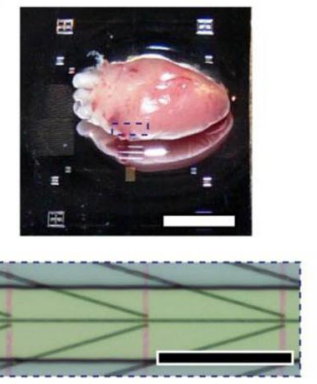

C

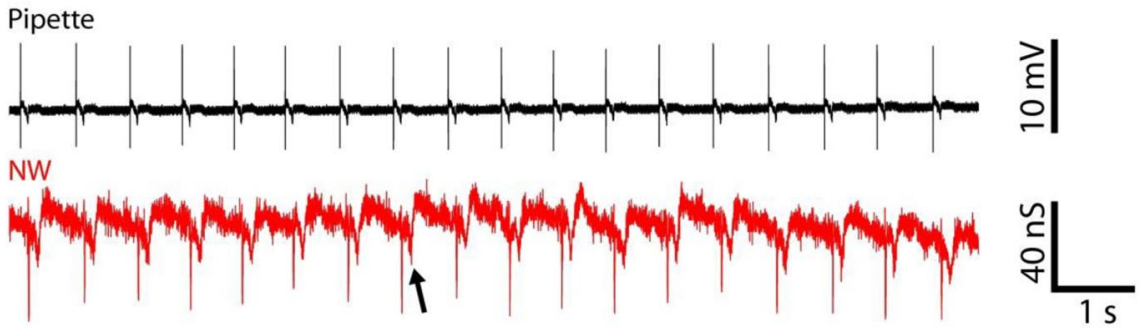

Fig. 3 NW/heart interfaces. (A) Photograph of experimental set-up showing heart on NW FET chip in temperatureregulated cell. Arrows show position of heart (red), $\mathrm{Ag} / \mathrm{AgCl}$ reference electrode (yellow), and source/drain interconnect wires (blue). (B) (top) Magnified image of heart on surface of planar chip; scale bar is $4 \mathrm{~mm}$. (bottom) Zoom of dotted region in upper image showing three pairs of NW FETs; scale bar is $150 \mu \mathrm{m}$. (C) Simultaneous recordings from a glass pipette (black trace) and an NW device (red trace). Adapted from Timko et al., American Chemical Society, copyright (C) 2009.

\section{Transparent and flexible substrates}

NWs and CNT FETs can be fabricated on flexible plastic substrates [38-40] and thus open up the possibility of making chips that can be readily deformed to tissue and organs or used for in vivo studies. We have explored this concept by assembling active NW FETs on 50- $\mu$ m-thick flexible and transparent Kapton substrates. These flexible and transparent NW FET chips enable simultaneous optical imaging and electronic recording in configurations that are not readily accessible with traditional planar device chips, yet advantageous for producing diverse, functional tissue-device interfaces. A bent device chip with concave surface facing a beating heart immersed in medium (Fig. 4A) illustrates this capability. We note that the chip is readily integrated into an upright microscope and allows for both visual inspection and higher-resolution imaging through the transparent substrate while recording from NW FET devices (Fig. 4B). Our capacity for simultaneous imaging of tissue and devices enables their registration at the level of the entire organ down to individual cells. Notably, recording from a representative NW FET device in this inverted configuration (Fig. 4C) demonstrated excellent S/N peaks correlated with the spontaneously beating heart. The average magnitude of the conductance peaks, $164 \pm 7 \mathrm{nS}$, and calibrated voltage, $4.5 \pm 0.2 \mathrm{mV}$, are similar to that recorded in more traditional "planar" configuration. In addition, similar recording was achieved on beating hearts in which bent chips were oriented with convex NW FET surface wrapped partially around the heart. Taken together, these results demonstrate that our flexible and transparent NW chips can be used to record electronic signals from organs in configurations not achievable by conventional electronics [37].

Our studies of neurons [41] and heart tissue [37] demonstrate that NW devices can be used as a general platform to investigate electrogenic biological systems. A separate group also demonstrated that lithographically patterned top-down NW FETs can be used to record signals from rat cardiomyocytes 


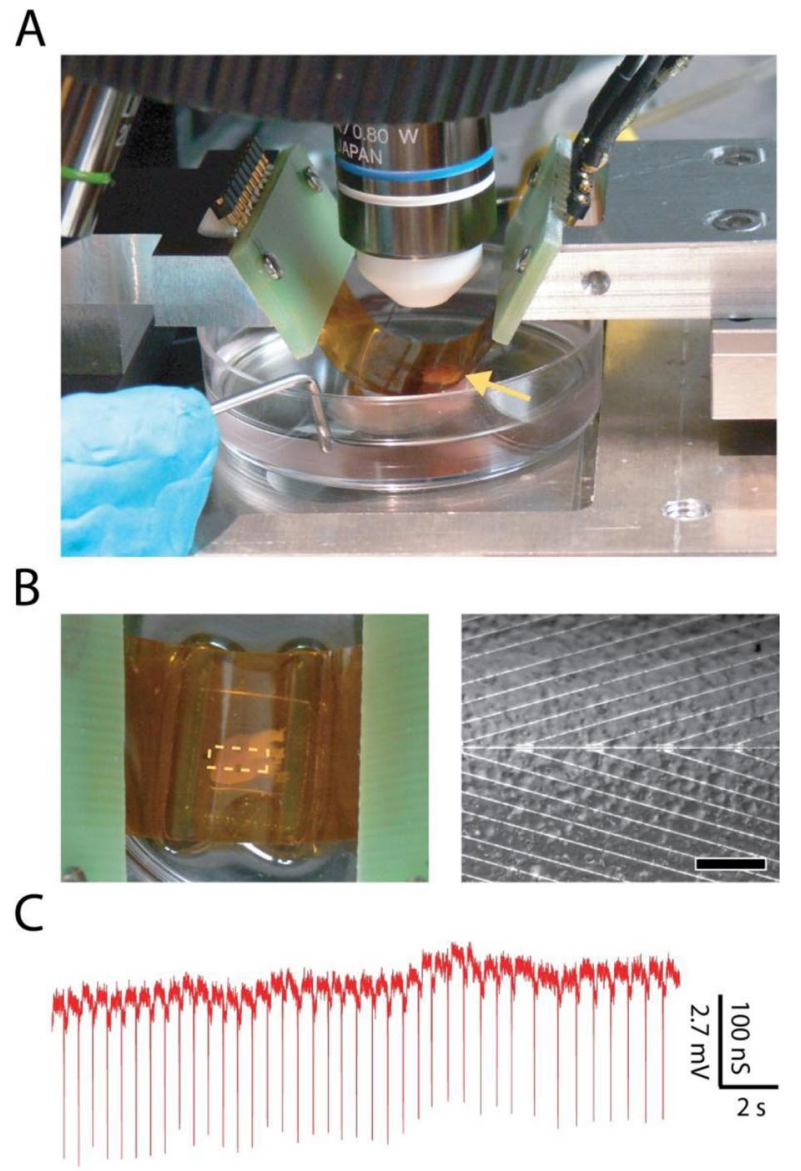

Fig. 4 NW FET recording in bent chip configuration. (A) Photograph of heart (yellow arrow) located underneath bent substrate with NW FETs on the lower concave face of the substrate. (B) (left) Top-down photograph of same system, which enables overall registration between heart and lithographically defined markers on the substrate. (right) Optical image taken with same system showing features on the heart surface vs. position of individual NW devices, which are located along the central horizontal axis. Scale bar is $150 \mu \mathrm{m}$. (C) Recorded conductance data from an NW FET in the configuration shown in panel a. Adapted from Timko et al., American Chemical Society, copyright (C) 2009.

and monolayers of rat aortic smooth muscle cells with high $\mathrm{S} / \mathrm{N}$ and $\mathrm{mV}$ amplitudes [42]. In our own laboratory, we investigated cultured cardiomyocyte monolayers in a new manner and probed the relationship between interfaces and signal magnitude [43]. This relationship, though never studied in the context of NW devices, is critical to understand given the unique interactions that exist between cells and nanoscale structures [26-34].

\section{FLEXIBLE INTERFACES WITH CELLS USING NW FETS}

\section{Characterization of NW/cardiomyocyte interfaces}

We developed a flexible scheme for interfacing cardiomyocytes and cells in general with NW FETs. Chips were fabricated using the same process used for neuron and heart studies [37,41] (Fig. 5A). Separately, cardiomyocytes were cultured on thin, optically transparent, and flexible pieces of poly- 
A

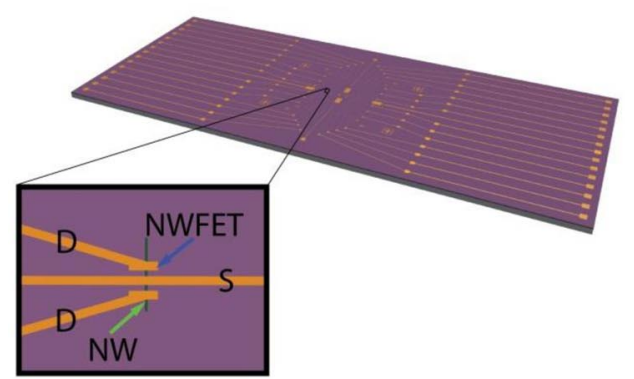

B

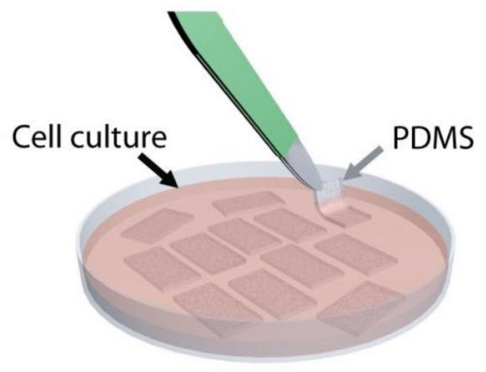

C

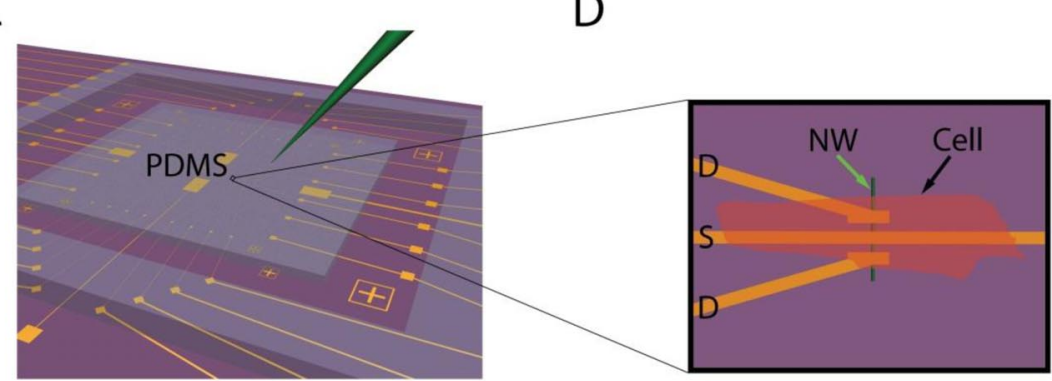

Fig. $5 \mathrm{NW/cardiomyocytes} \mathrm{interfaces.} \mathrm{(A)} \mathrm{Schematics} \mathrm{of} \mathrm{the} \mathrm{experimental} \mathrm{approach.} \mathrm{NW} \mathrm{FET} \mathrm{chip,} \mathrm{where} \mathrm{NW}$ devices are located at the central region of chip. The visible linear features (gold) correspond to NW contacts and interconnect metal. Zoom-in showing a source (S) and two drain (D) electrodes connected to a vertically oriented NW (blue arrow) define two NW FETs. (B) Cardiomyocytes cultured on thin flexible pieces of PDMS, where (green) one piece is being removed with tweezers. (C) PDMS substrate with cultured cells oriented over the device region of the NW FET chip. The green needle-like structure indicates the probe used to both manipulate the PDMS/cell substrate to specific NW device locations. (D) Schematic of (black arrow) a cardiomyocyte oriented over (green arrow) an NW device. Adapted from Cohen-Karni et al., National Academy of Sciences, copyright (C) 2009.

dimethylsiloxane (PDMS) (Fig. 5B) to form cell monolayers, and then a PDMS/cardiomyocyte substrate was positioned over the NW FET chip in extracellular medium (Fig. 5C). PDMS/cardiomyocyte cell substrates were aligned to bring spontaneously beating cells into direct contact with the NW FETs (Fig. 5D), thus enabling measurements from defined monolayer regions with specific devices.

The ability to manipulate the PDMS/cell substrate independent of the NW FET chip also enables us to identify specific cardiomyocyte regions using an optical microscope, place the desired cell or cell network over NW FET devices, and then record from the desired region. Two examples are shown in Fig. 6. In the first case, a patch of spontaneously beating cells was located (red dashed line, Fig. 6A), placed over a specific device, and conductance vs. time data recorded (Fig. 6B) yielded a signal amplitude of $4.2 \pm 0.3 \mathrm{mV}$. In a second example, a distinct cell in a cardiomyocyte monolayer was located (red dashed line, Fig. 6C), placed over a different device and conductance vs. time data yielded an amplitude of $2.1 \pm 0.3 \mathrm{mV}$ (Fig. 6D). Importantly, our ability to identify and register specific cellular regions over NW FET elements has not been demonstrated previously for either planar or nanoscale FET where cells have been cultured directly over device chips. This capability opens up the possibility of a number of interesting studies in the future, including multiplexed recording from well-defined multi-cellular configurations as well as multiplexed measurements at the single cell level for subcellular resolution investigations. 
A

\section{B}
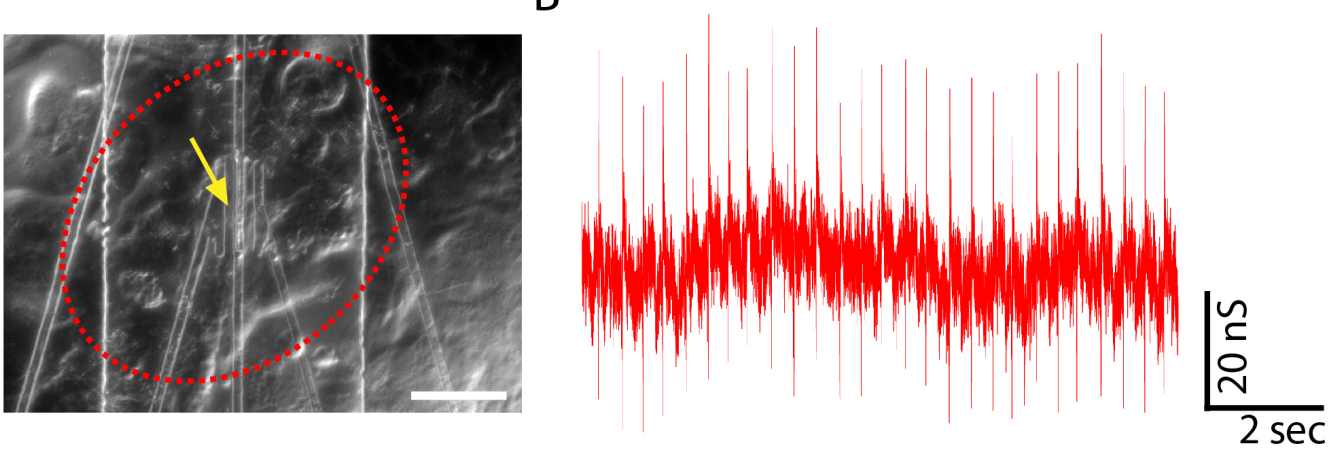

C
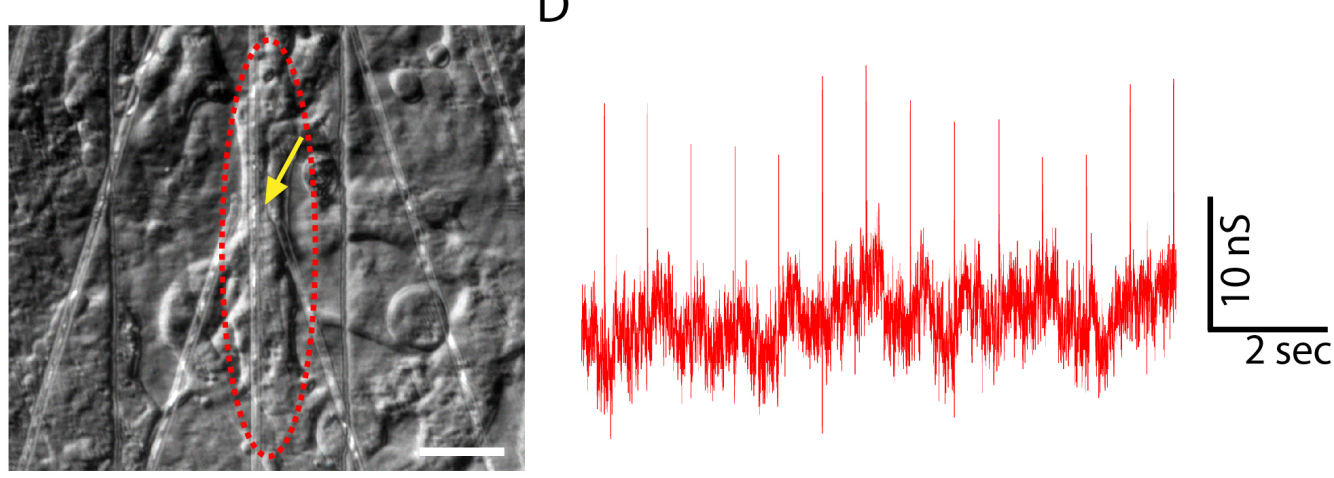

Fig. 6 Recording from distinct regions of cardiomyocytes monolayers. (A) A patch of beating cells (red dashed oval) over an NW FET (yellow arrow); scale bar is $40 \mu \mathrm{m}$. (B) Conductance vs. time signals recorded from this cell patch. (C) Distinct patch of beating cells (red dashed oval) over an NW device (yellow arrow); scale bar is 20 $\mu \mathrm{m}$. (D) Conductance vs. time signals recorded from the cells. Adapted from Cohen-Karni et al., Proc. Natl. Acad. Sci. USA, copyright (C) 2009.

These basic results for NW FET recording from PDMS/cardiomyocyte samples can be compared to previous studies of cardiomyocytes. For example, studies of cardiomyocyte monolayers cultured on conventional planar FET devices have yielded peaks with $\mathrm{S} / \mathrm{N}$ of 2-6 and amplitudes from 0.2 to $2.5 \mathrm{mV}[7,8]$. In general, our NW FETs yield better $\mathrm{S} / \mathrm{N}$ with values $>4$ and a maximum of 25 observed. In addition, the typical calibrated voltages recorded in our measurements are similar to or greater than the largest values reported previously. The improvement in peak amplitude is consistent with the fact that the nanodevices protrude from the plane of substrate and hence can increase NW/cell interfacial coupling [26-34].

\section{NW sensitivity experiments}

These key features of our experiment can be seen in the photograph of a typical set-up (Fig. 7A), which highlights the NW FET chip with flexible input/output to recording instruments, PDMS/cell substrate manipulator, and microscope objective. Plots of NW FET conductance $(G)$ vs. applied water-gate voltage $\left(V_{\mathrm{g}}\right)$ for three representative devices immersed in extracellular medium yield sensitivity values, $G / V_{\mathrm{g}}$, at $V_{\mathrm{g}}=-0.3 \mathrm{~V}$ of $13.8,17.2$, and $31.1 \mathrm{nS} / \mathrm{mV}$. These sensitivities are similar to our previous studies of NW FET devices fabricated on both rigid and flexible plastic substrates [21]. In addition, optical images (inset, Fig. 7B) show clearly that individual devices can be visualized, including S/D electrodes and the NW active element. 
A

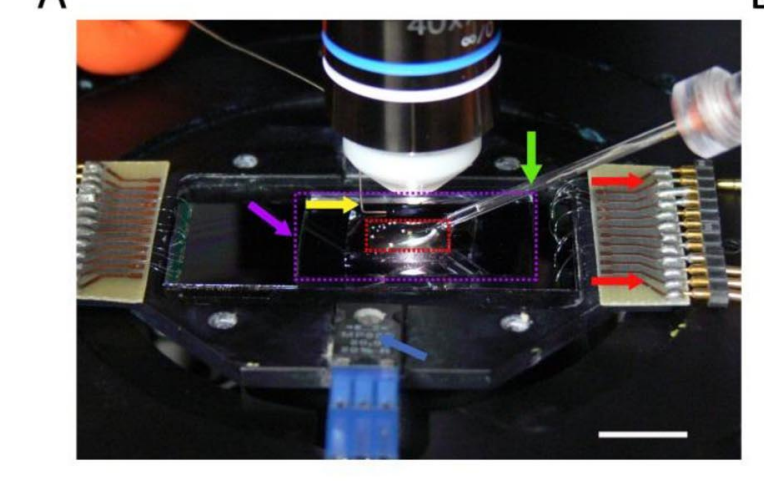

B
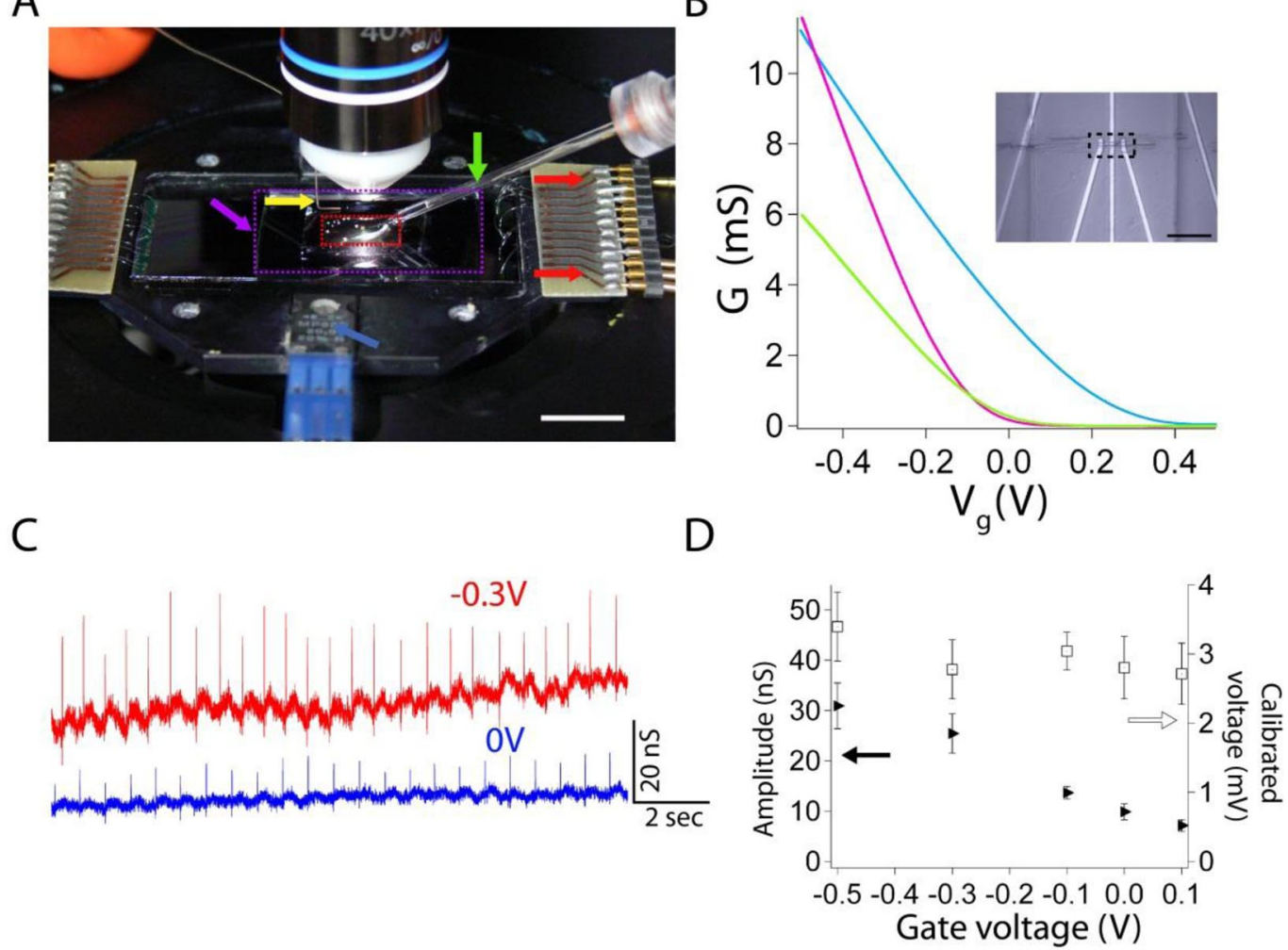

Fig. 7 Measurement of cardiomyocyte signals. (A) Photograph of the experimental set-up showing the PDMS piece (red dashed box) on top of an NW FET chip within a solution well that is temperature-regulated with an integrated heater (blue arrow). Additional yellow, purple, green, and red arrows highlight positions of the $\mathrm{Ag} / \mathrm{AgCl}$ reference electrode, solution medium well (length $\times$ width $\times$ depth $=25-30 \times 15-20 \times 2 \mathrm{~mm}^{3}$ ), glass manipulator/force pipette connected to $\mathrm{x}-\mathrm{y}-\mathrm{z}$ manipulator, and plug-in connectors between NW FET interconnect wires and measurement electronics, respectively. Scale bar is $10 \mathrm{~mm}$. (B) Representative gate responses of three SiNW FET devices. The sensitivity $\left(G / V_{\mathrm{g}}\right)$ of separate devices represented by the green, cyan, and purple traces at $V_{\mathrm{g}}=-0.3 \mathrm{~V}$ are $13.8,17.2$, and $31.1 \mathrm{nS} / \mathrm{mV}$, respectively. Inset shows optical microscopy image of two NW FET devices (dashed box) as illustrated schematically in Fig. 1. Scale bar is $20 \mu \mathrm{m}$. (C) Conductance vs. time traces recorded at $V_{\mathrm{g}}=-0.3 \mathrm{~V}$ (red) and $0 \mathrm{~V}$ (blue) for the same NW FET-cardiomyocyte interface; the device sensitivities at -0.3 and $0 \mathrm{~V}$ were 9.2 and $3.5 \mathrm{nS} / \mathrm{mV}$, respectively. (D) Plots of peak conductance amplitude (full triangles) and calibrated peak voltage amplitude (open squares) vs. $V_{\mathrm{g}}$; data was obtained from the same experiments shown in (C). Error bars correspond to \pm 1 standard deviation (SD). Adapted from Cohen-Karni et al., Proc. Natl. Acad. Sci. USA, copyright @ 2009.

Measurement of the conductance vs. time from a SiNW FET in contact with a spontaneously beating cardiomyocyte cell monolayer (Fig. 7C) yields regularly spaced peaks with a frequency of ca. $1.5 \mathrm{~Hz}$ and $\mathrm{S} / \mathrm{N}, \geq 4$. Comparison of the two traces (Fig. 7C) also shows that the conductance peak magnitude is directly related to the device sensitivity; that is, the data recorded at $V_{\mathrm{g}}=-0.3$ and $0 \mathrm{~V}$ had average peak amplitudes of $25.4 \pm 3.9$ and $9.9 \pm 1.6 \mathrm{nS}$, respectively. Notably, the calibrated voltages for these traces, $2.8 \pm 0.4$ and $2.8 \pm 0.5 \mathrm{mV}$, were the same within experimental uncertainty. These results confirm the stability of the interface between the NW FETs and PDMS/cardiomyocyte cells, and highlight the necessity of recording explicit device sensitivity to interpret corresponding voltages. This important point is further illustrated in the summary of data recorded with $V_{\mathrm{g}}$ values from -0.5 to $0.1 \mathrm{~V}$ 
(Fig. 7D), where the conductance signal amplitudes decrease from 31 to $7 \mathrm{nS}$, respectively, but the calibrated voltage, $2.9 \pm 0.3 \mathrm{mV}$, remained unchanged [43].

\section{Cell substrate displacement experiments}

The ability to manipulate the PDMS/cell substrate independent of the NW FET chip also opens up new opportunities compared with cells cultured directly on device arrays. For example, we investigated the relationship between recorded signal magnitude when a micropipette displaced the PDMS a fixed distance (Fig. 8A). A direct comparison of single peaks recorded for increasing displacement values (Fig. 8B) shows a consistent monotonic increase in peak amplitudes from 31 to $72 \mathrm{nS}$. In addition, this high-resolution peak comparison demonstrates that there is no observable change in peak shape or peak width over this $>2 \times$ change in amplitude, and that the peak width is consistent with time-scales for ion fluxes associated with ion-channels opening/closing [7]. A summary of data obtained for increasing/decreasing displacements (Fig. 8C) shows clearly the systematic 2.3 -fold increase in peak amplitude, and moreover, demonstrates these amplitude changes are reversible. Recent studies of Aplysia neurons cultured on planar FET devices have also reported an increase in peak amplitude when the cell body was displaced [44]. In both cases, the enhanced signal amplitudes can be attributed to a

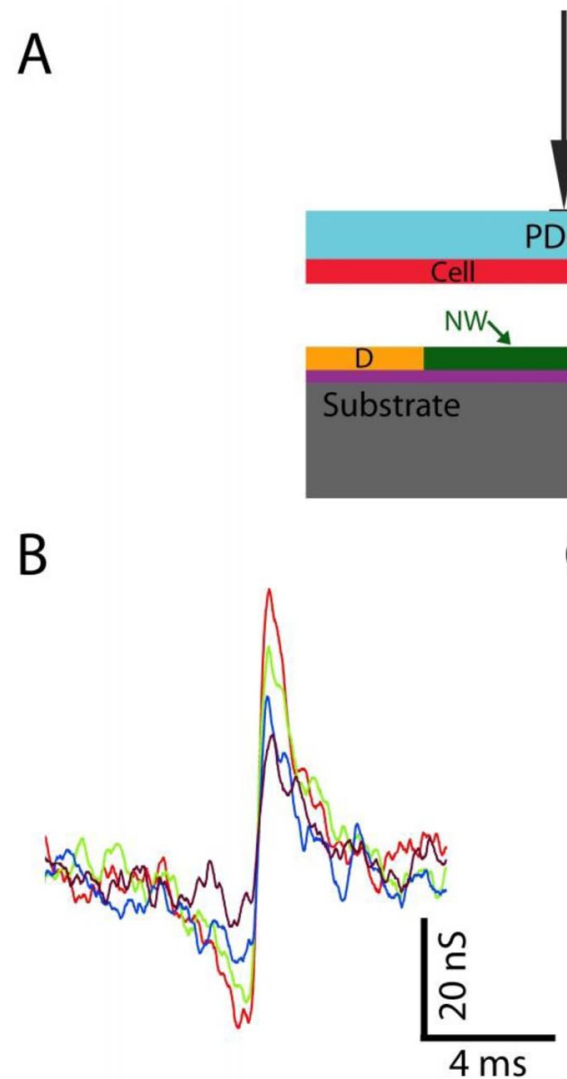

Z
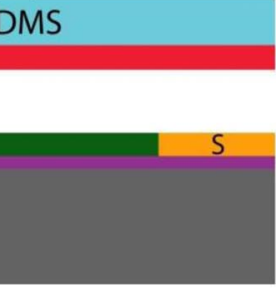

C

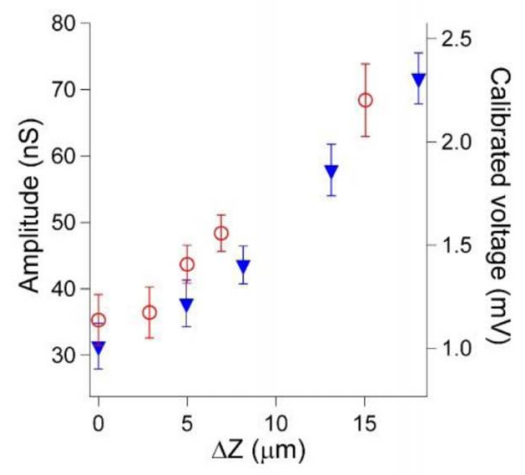

Fig. 8 Effect of applied force on recorded signals. (A) Schematic illustrating displacement (Z) of the PDMS/cell substrate with respect to an NW FET device. (B) High-resolution comparison of single peaks recorded with $\Delta \mathrm{Z}$ values of $0 \mu \mathrm{m}$ (purple), $8.2 \mu \mathrm{m}$ (blue), $13.1 \mu \mathrm{m}$ (green), and $18.0 \mu \mathrm{m}$ (red). (C) Summary of the recorded conductance signals and calibrated voltages vs. $\Delta \mathrm{Z}$, where the open red circles (filled blue triangles) were recorded for increasing (decreasing) $\Delta$ Z. Adapted from Cohen-Karni et al., Proc. Natl. Acad. Sci. USA, copyright (C) 2009. 
decrease in gap between the cell membrane and devices, although future studies will be needed to quantify such junction changes.

\section{ULTRA-SHORT-CHANNEL NW TRANSISTORS FOR POINT-LIKE CELLULAR INTERFACES}

\section{NW synthesis and investigations}

Although most SiNW systems are synthesized by the well-studied vapor-liquid-solid (VLS) growth mechanism, it was recently shown $[45,46]$ that a vapor-solid-solid (VSS) mechanism can be used to form abrupt $\mathrm{Si} / \mathrm{Ge}$ heterojunctions along the growth direction of the NWs. The abrupt junctions were made possible due to slow growth rates of the VSS mechanism, which are at least 10-100 times lower than for VLS grown NWs [47-49]. Although the VSS growth mechanism has been demonstrated, a viable method to synthesize functional "point-like" NW devices by controlling the dopant profile has not been realized. We reported the first bio-electronic interface between cells and synthetically integrated short-channel SiNW FETs grown by a VSS mechanism. The flexibility of the bottom-up approach has enabled us to explore the size scale at which electrical extracellular interfaces can be made.

Our approach to synthesize SiNWs with controlled NW dopant profiles is presented in Fig. 9A. First, we synthesize a highly doped n-type $\left(\mathrm{n}^{++}\right)$segment that will serve as the source (S) electrode using the VLS mechanism at a temperature above the Au-Si eutectic point $\left(T_{\mathrm{eu}}\right)$. Second, in order to synthesize the short channel we reduce the temperature below $T_{\text {eu }}$ to solidify the Au catalyst and transition to a VSS growth mechanism, which permits superior control of the channel length and dopant profile as compared to VLS growth mechanism [49]. Last, we raise the temperature above $T_{\text {eu }}$ to transition back to a VLS growth mechanism and synthesize the last $\mathrm{n}^{++}$region, which will serve as the drain (D) electrode for the short-channel device. As illustrated in Fig. 10A, these short-channel devices are used to create a cellular interface potentially capable of detection on the length scale of a few protein molecules or ion channels [50].

The key synthetic parameters for the SiNW short-channel devices are characterized in Fig. 9. We synthesized NWs with channel lengths of 50, 80, and $150 \mathrm{~nm}$, as exemplified by the scanning electron microscopy (SEM) images of selectively etched NWs (Fig. 9B). Using a calibrated growth rate of $1.0 \mathrm{~nm} / \mathrm{min}$ (Fig. 9C), these three $\mathrm{n}^{++} / \mathrm{i} / \mathrm{n}^{++}$structures were grown in VSS mode at $340{ }^{\circ} \mathrm{C}$ by changing the growth time used for the intrinsic channel. Our growth rates are in good agreement with published data $[45,46]$ and are $~ 10-100$ times smaller than published VLS growth rates of $100-600 \mathrm{~nm} / \mathrm{min}$ $[47,48]$. In addition, phosphorous elemental mapping obtained with an aberration-corrected scanning transmission electron microscope (Cs-STEM) allowed us to quantitatively characterize the abruptness of the $\mathrm{n}^{++} / \mathrm{i} / \mathrm{n}^{++}$dopant transition. As shown in Fig. 9D, the elemental map and line profile exhibit an abrupt drop in phosphorus counts over a span of $\sim 5 \mathrm{~nm}$ within the intrinsic channel of the NW. These results are in accord with previously synthesized heterostructures using a VSS growth mode $[45,46]$ and demonstrate our unique capability to synthetically encode sharp, well-defined dopant junctions in the NWs. 


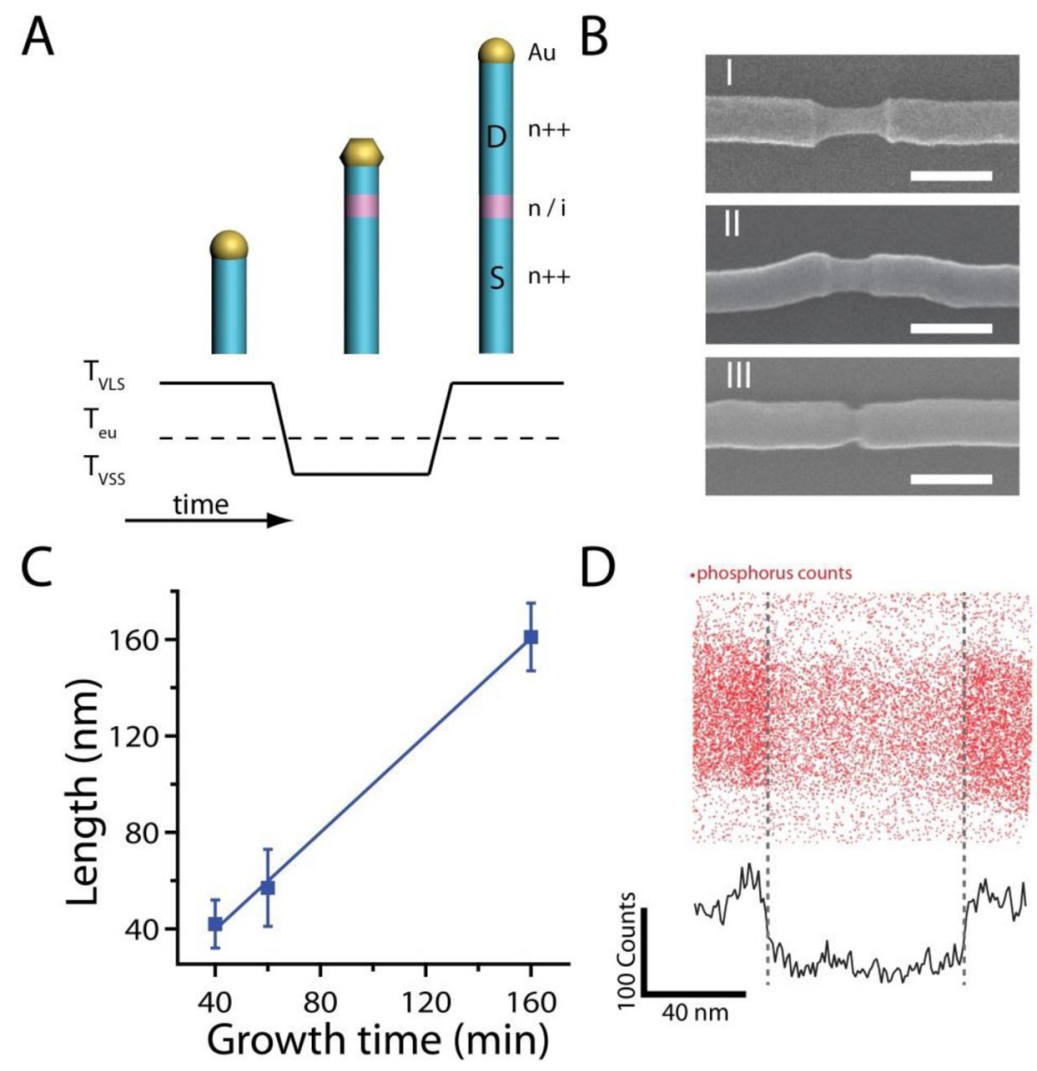

Fig. 9 Short-channel SiNW synthesis. (A) Overview of the synthesis and cellular interfaces of short-channel NW FETs. Illustration of gold-nanocluster-catalyzed NW growth with well-controlled axial dopant profile introduced during VSS growth. Initially, a ${ }^{++} \mathrm{S}$ electrode is synthesized via the VLS mechanism. Subsequently, either $\mathrm{n}$ or $\mathrm{i}$ active device regions are encoded by VSS growth. Lastly, another VLS phase of growth completes the $n^{++} \mathrm{D}$ electrode. (B) Short-channel $\mathrm{n}^{++} / \mathrm{i} / \mathrm{n}^{++}$SiNWs with channel lengths of $150 \mathrm{~nm}$ (I), $80 \mathrm{~nm}$ (II), and $50 \mathrm{~nm}$ (III) using growth times of 160,80 , and $40 \mathrm{~min}$, respectively, at a VSS growth temperature of $340{ }^{\circ} \mathrm{C}$. Scale bars are $150 \mathrm{~nm}$. The gold nanoclusters were $\sim 80 \mathrm{~nm}$ in diameter, and NWs were selectively etched to reveal the active channel. (C) Dependence of the channel length on VSS growth time at $340{ }^{\circ} \mathrm{C}$. Values are average \pm SD (calculated for 20 SiNWs per growth time.) (D) Energy-dispersive X-ray (EDX) elemental mapping of $\mathrm{P}$ dopant in an $\mathrm{n}^{++} / \mathrm{i} / \mathrm{n}^{++} \mathrm{NW}$, showing a spatial map of individual P X-ray counts (top) and line profile of P counts (bottom), generated by radial integration of the $\mathrm{P}$ counts shown in the top panel. Adapted from Cohen-Karni et al., American Chemical Society, copyright (C) 2012.

\section{Cellular interfaces}

Following the synthesis, we fabricated FET devices from the short-channel NWs. Briefly, as-synthesized SiNWs were deposited from isopropanol solution on a $\mathrm{Si}$ substrate coated with $50 \mathrm{~nm} \mathrm{Si}_{3} \mathrm{~N}_{4}$, and metal interconnects to the S/D were defined using electron beam lithography (EBL). The metal was then passivated with SU8 and/or poly(methyl methacrylate) (PMMA). We verified the performance of the short-channel NWs by measuring conductance under an applied water-gate potential (Fig. 10B) and measured sensitivities of 13.5, 21.0, and $6.4 \mathrm{nS} / \mathrm{mV}$ for channel lengths of $150 \mathrm{~nm}$ (case I), $80 \mathrm{~nm}$ (case II), and $50 \mathrm{~nm}$ (case III). Following the electrical characterization, these devices were interfaced with spontaneously beating embryonic chicken cardiomyocytes as previously described [43], and measurement of conductance vs. time yielded regularly spaced peaks with a frequency of $1.1-1.3 \mathrm{~Hz}$ (Fig. 10C). All three cases recorded the rhythmic behavior of the cells and showed fairly good S/N (>2). Notably, 
A

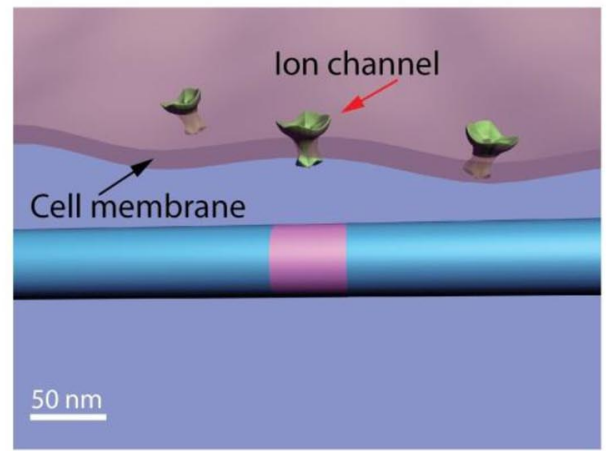

C

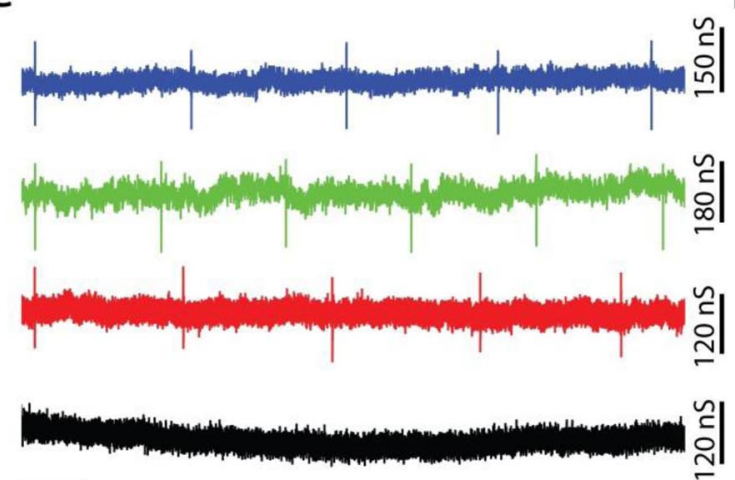

$\overline{400 \mathrm{~ms}}$
B

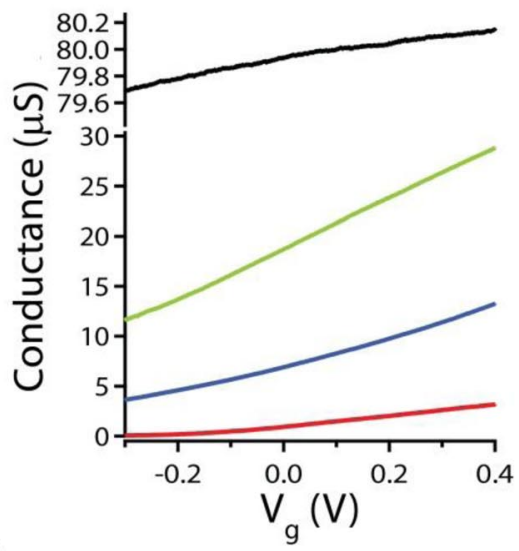

D

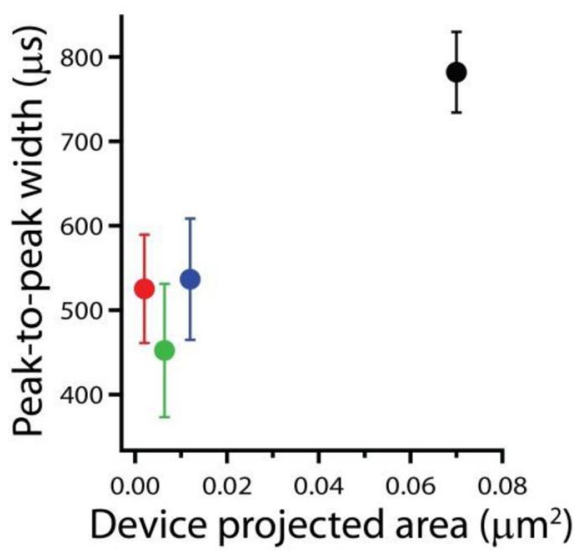

Fig. 10 Short-channel SiNW FETs interfaced with cardiomyocytes. (A) Schematic of a short-channel NW FET interfaced with an extracellular region of an electrogenic cell. (B) Conductance of NW FETs as a function of watergate potential $\left(V_{\mathrm{g}}\right)$ for channel lengths of $150 \mathrm{~nm}$ (i, blue), $80 \mathrm{~nm}$ (i, green), and $50 \mathrm{~nm}$ (n, red). Black trace is a control device fabricated on an $\mathrm{n}^{++}$segment without an active channel. (C) Typical recorded signals from beating cardiomyocytes for devices presented in panel A. The $\mathrm{n}^{++}$control (black trace) was recorded simultaneously with the 80-nm channel-length device. For the 50-nm channel-length device, a 40-nm-diameter NW was used, whereas the other NW devices were $80 \mathrm{~nm}$ in diameter. For the 150- and 80-nm channel-length devices, $V_{\mathrm{g}}=0 \mathrm{~V}$, and for the 50-nm channel-length device, $V_{\mathrm{g}}=+0.3 \mathrm{~V}$. (D) Summary of the peak-to-peak widths for the each of the shortchannel structures. In addition, a previously published 2.3- $\mu$ m channel-length SiNW device (black) is shown for comparison. Adapted from Cohen-Karni et al., American Chemical Society, copyright (C) 2012.

the calibrated voltages of these peaks are the largest amplitudes yet recorded using SiNW FETs, yielding values of 14.4, 12.5, and $25.7 \mathrm{mV}$ for cases I, II, and III, respectively. To prove that only the active channel records signals, we simultaneously recorded a control device fabricated on one of the $\mathrm{n}^{++}$arms; not surprisingly, the short-channel device recorded rhythmic changes in conductance while the control device showed no signal (Fig. 10C black trace). Further investigation of the peak-to-peak width of the recorded signals (Fig. 10D) indicates that these signals have a peak-to-peak width of $520 \pm 40,450 \pm$ 80 , and $540 \pm 50 \mu$ s for cases I, I, and III, respectively. These results are significantly smaller than the peak-to-peak width, 750-850 $\mu$ s, measured for devices with micron-scale active channels [43,51] (Fig. $10 \mathrm{D}$, purple circle). Interestingly, the time constants reported for sodium ion channel conduction are ca. $500 \mu \mathrm{s}$ [52], which is in good accord with time constants measured from short-channel NW FETs. The 
results indicate that these NW devices may be capable of measuring ion channel activity on the length and time scale of single ion channel events; however, further studies will be necessary to corroborate this hypothesis.

\section{Multiplexed measurements}

To further illustrate the capabilities of this new approach, we reproducibly synthesized multiple $\mathrm{n}^{++} / \mathrm{i} / \mathrm{n}^{++}$channels on a single SiNW either via VSS or VLS growth mode [50]. We utilized the flexibility of this bottom-up approach to fabricate closely spaced FETs on single NWs and interfaced these devices with cardiomyocytes (Figs. 11A-C), giving rise to clean signals at a $\sim 1 \mathrm{~Hz}$ frequency (Fig. 11D). Quantitative analysis of the time lags between devices is presented in Fig. 11E [50]. The signal from device 1 (labeled d1 in Fig. 11C) precedes device 2 (d2) and device 3 (d3) because of spatial propagation of the signal from d1 toward $\mathrm{d} 3$. Moreover, the signal spreads from $\mathrm{d} 1$ to both $\mathrm{d} 2$ and $\mathrm{d} 3$ with speeds of 0.17 and $0.35 \mathrm{~m} / \mathrm{s}$.

In addition, we have extended our approach of synthetically encoding devices to separations smaller than $2 \mu \mathrm{m}$ (Fig. 11F). Specifically, three 130-nm short-channel devices were interfaced with spontaneously beating cardiomyocytes and used to record the conductance changes as a function of time (Fig. 11G). These data show well-defined, correlated extracellular peaks with a $\sim 1 \mathrm{~Hz}$ frequency. Data from 375 beating events recorded simultaneously from the three devices were analyzed [41] to determine time differences between pairs of devices. The results (Fig. 11H) show time lags of 4.9 and $89 \mu$ s for the two devices separated by $1.9 \mu \mathrm{m}$ (Fig. 11G, d1 and d2) and $73 \mu \mathrm{m}$ (Fig. 11G, d1 and d3), respectively. The corresponding signal propagation speeds of 0.4 and $0.8 \mathrm{~m} / \mathrm{s}$ are in good agreement with published data of signal transduction in cultured cardiomyocytes. Interestingly, the signal propagation across multiple cell junctions, between $\mathrm{d} 3$ to $\mathrm{d} 1$, showed a larger SD $(19 \mu \mathrm{s})$ than the case of within a single cell (d2 to d1, 7.6 $\mu \mathrm{s}$ ). These results may reflect multiple signal paths over longer distances between cells, although future studies will be required to conclusively illuminate the nontrivial deviations in the distributions, including, for example, the dynamic redistribution of density of ion channels over the time course of our recordings [53].

In conclusion, we have demonstrated the synthesis of axial dopant-modulated ultrashort SiNW FET devices via the VSS growth method and the use of these devices for recording extracellular field potentials with high spatial resolution. Using the VSS mode, we were able to grow segments as small as $50 \mathrm{~nm}$ in 40-nm-diameter SiNWs. Elemental mapping of phosphorous across the short-channel segments revealed a transition length $<5 \mathrm{~nm}$, which is an order of magnitude smaller than the VLS transition length. Devices with 150-, 80-, and 50-nm channel lengths faithfully recorded extracellular field potentials from beating cardiomyocytes and demonstrated no decrease in the calibrated (voltage) extracellular potentials and $\mathrm{S} / \mathrm{N}$ with decreasing device size. Temporal analysis of the recorded peaks also revealed distinct differences between these ultrashort devices and longer channel-length devices. The peak-to-peak width of the ultrashort devices, $\sim 500 \mu \mathrm{s}$, was comparable to the intrinsic time constant for $\mathrm{Na}^{+}$-ion channels and smaller than longer channel-length devices, thus highlighting the potential of these "point-like" detectors for probing ion channel activity. Moreover, the flexibility of the bottom-up synthetic approach allowed us to create multiple ultrashort devices in single SiNWs, allowing us to detect signal propagation at the subcellular level. These findings open up unique opportunities for fundamental, subcellular biophysical studies and also make steps toward the limit of building electronic interfaces at close to the molecular level. 
A

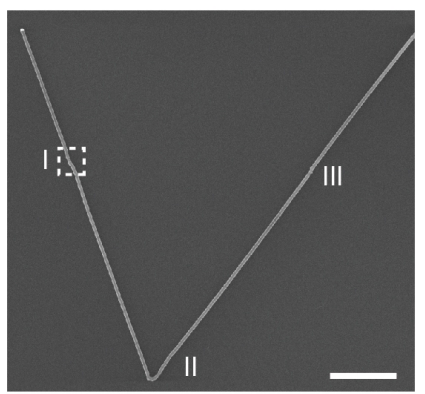

D
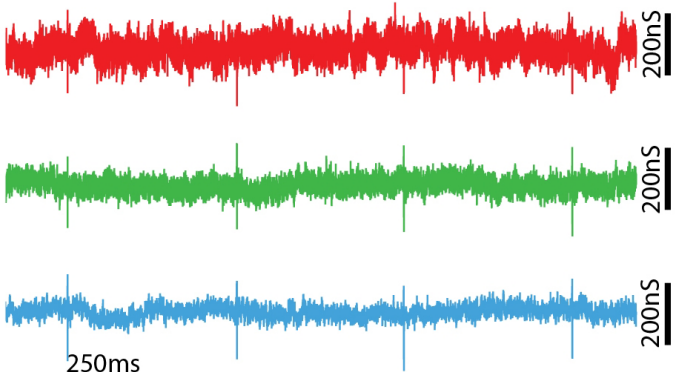
$250 \mathrm{~ms}$

$\mathrm{F}$
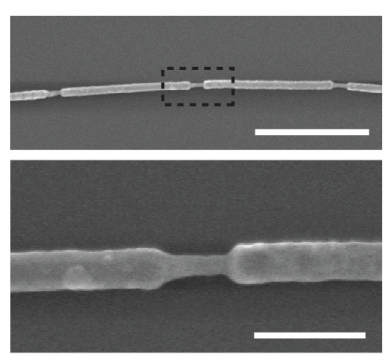

G

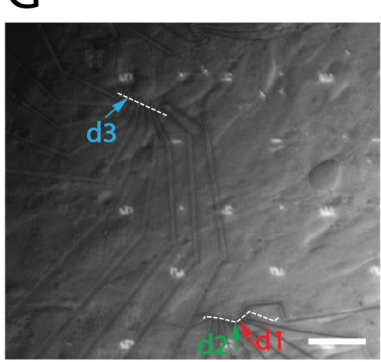

B

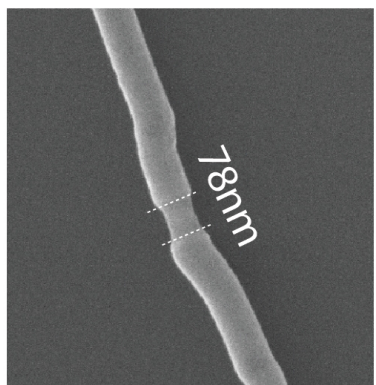

$E$

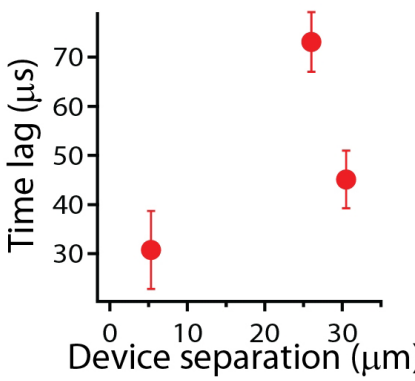

$\mathrm{H}$

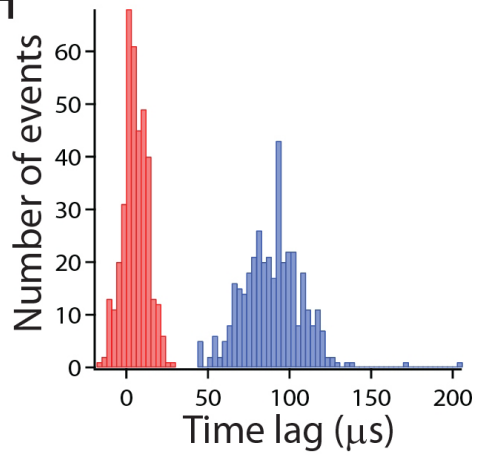

Fig. 11 Multiplexed recording with short-channel devices. (A) SEM image of a representative 80-nm SiNW with three $80-\mathrm{nm}$ channel-length devices. Scale bar is $1.5 \mu \mathrm{m}$. (B) An expanded view of the short-channel segments marked with white dashed box (C) Optical image of three 80-nm short-channel SiNW devices interfaced with cardiomyocytes. The first and second devices (d1 and d2) are on the same NW, and the third device (d3) is on a separate NW (white dashed lines highlight the NW positions). Scale bar is $15 \mu \mathrm{m}$. (D) Representative conductance vs. time signals from $\mathrm{d} 1$ (red), $\mathrm{d} 2$ (green), and $\mathrm{d} 3$ (cyan). (E) Time lag between the three devices as determined by correlation analysis (values are average $\pm \mathrm{SD}$, calculated from 58 beating events). The distance between devices was $5.3 \mu \mathrm{m}$ for $\mathrm{d} 1-\mathrm{d} 2,26 \mu \mathrm{m}$ for $\mathrm{d} 1-\mathrm{d} 3$, and $30.5 \mu \mathrm{m}$ for $\mathrm{d} 2-\mathrm{d} 3$. (F) Recording from short-channel SiNW FETs on multiple length scales. Upper panel: SEM image of an 80-nm-diameter NW encoded with 130-nm channel lengths that are separated by $1.1 \mu \mathrm{m}$; scale bar is $1 \mu \mathrm{m}$. Lower panel: An expanded view of the segment marked with a black dashed box; scale bar is $200 \mathrm{~nm}$. (G) Optical image of cardiomyocytes interfaced with three 130-nm channel-length devices (labeled d1, d2, and d3). White dashed lines illustrate the NW position; scale bar is $15 \mu \mathrm{m}$. (H) Histogram of the time lag between devices $\mathrm{d} 1$ and $\mathrm{d} 2$ (red; separation distance $1.9 \mu \mathrm{m}$ ) and between devices d1 and $\mathrm{d} 3$ (blue; separation distance $73 \mu \mathrm{m}$ ). Adapted from Cohen-Karni et al., American Chemical Society, copyright $\odot 2012$. 


\section{CONCLUSION AND PROSPECTS}

In recent years, NW devices have been implemented as a broad platform for electronic interfaces with cells and tissue, demonstrating several key points [37,41,43,51,54]. First, NW FETs having orders of magnitude smaller recording area than previous techniques were used to measure extracellular signals from individual cells and tissue. These signals, in the $\mathrm{mV}$ range, are considerably larger than those measured using planar, lithographically patterned FETs or MEAs [5-8], possibly because of enhanced coupling between the nanoscale device and cell membrane. The signal magnitude is directly correlated to (a) device sensitivity (transconductance) $[37,43]$ and (b) interface quality between cells and substrate [43]. Second, NW devices were used to connect with individual neurons at the level of individual axons or dendrites [41], and with heart tissue [37] or cardiomyocytes at the subcellular level [43,50]. Moreover, multiplexed recording from NW FETs has subsequently enabled signal mapping in the context of neurons, acute brain slices, heart tissue, and cardiomyocytes [37,41,43,54]. Third, using our well-controlled synthesis method and utilizing the advantages of the bottom-up approach we demonstrated for the first time recording of electrical signals within a cell using either an unconventional nanoFET 3D probe $[55,56]$ or new device geometries that break the planar natures of NW FETs fabricated on chip surface [57].

These methods represent a general and flexible approach for creating hybrid nanoelectronic-biological devices that serve as the foundation for new, fundamental studies as well as novel directions in biomedical research and applications (Fig. 12). A remarkable property of NWs is that their material composition and corresponding properties can be tuned at the time of synthesis. This opens up future opportunities in the design of ultra-high sensitivity transistors through band structure engineering $[13,16]$, incorporation of photonic materials for light-addressable interfaces with photoactive cells [58], or use of additional novel geometries (e.g., branched NWs [17,59]) to further improve device-membrane coupling [57]. Moreover, incorporation of different geometries and types of devices will enable a two-way communication with cells and tissue, i.e., the use of vertical NW electrode array or stealthy metal probes $[60,61]$ for stimulation and nanoFETs for recording of signals.

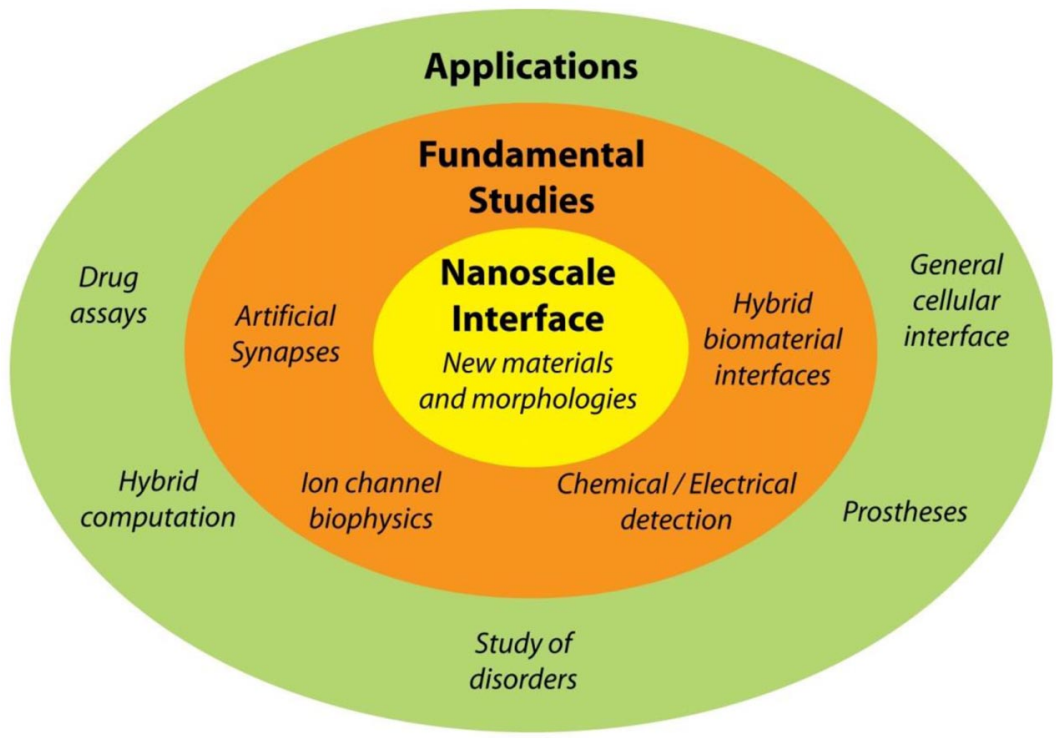

Fig 12 Overview of a bottom-up paradigm for NW nanobioelectronic interfaces. 
Finally, in the future, NW device arrays may be used to correlate electronic signaling with chemical release or to simultaneously detect a matrix of biologically relevant species. These techniques, pushing the boundaries of present technologies, could lead to new drug assays or breakthroughs in fundamental bioscience. NWs could also serve as a foundation for new and powerful prosthetic devices. Significantly, NW devices assembled on biocompatible substrates that form intimate extracellular or intracellular connections with excitable cells and tissue may be used as functional prosthetics, which could complement current biomedical technologies, by blurring the distinction of biological systems and electronic systems [62]. This new direction has been demonstrated recently by the first demonstration of a merging of digital world and a tissue in the form of an engineered tissue with embedded nanoelectronics motifs [63].

\section{REFERENCES}

1. R. D. Purves. Microelectrode Methods for Intracellular Recording and Ionophoresis, Biological Techniques series, Academic Press, London (1981).

2. B. Sakmann, E. Neher. Annu. Rev. Physiol. 46, 455 (1984).

3. I. R. Efimov, V. P. Nikolski, G. Salama. Circ. Res. 95, 21 (2004).

4. M. Scanziani, M. Hausser. Nature 461, 930 (2009).

5. M. Halbach, U. Egert, J. Hescheler, K. Banach. Cell. Physiol. Biochem. 13, 27 (2003).

6. M. Reppel, F. Pillekamp, Z. J. Lu, M. Halbach, K. Brockmeier, B. K. Fleischmann, J. Hescheler. J. Electrocardiol. 37, 104 (2004).

7. S. Ingebrandt, C. K. Yeung, M. Krause, A. Offenhausser. Biosens. Bioelectron. 16, 565 (2001).

8. C. K. Yeung, S. Ingebrandt, M. Krause, A. Offenhausser, W. Knoll. J. Pharmacol. Toxicol. Methods 45, 207 (2001).

9. D. J. Banks, W. Balachandran, P. R. Richards, D. Ewins. Physiol. Meas. 23, 437 (2002).

10. O. J. Prohaska, F. Olcaytug, P. Pfundner, H. Dragaun. IEEE Trans. Biomed. Eng. 33, 223 (1986).

11. A. M. Morales, C. M. Lieber. Science 279, 208 (1998).

12. J. T. Hu, T. W. Odom, C. M. Lieber. Acc. Chem. Res. 32, 435 (1999).

13. W. Lu, C. M. Lieber. J. Phys. D: Appl. Phys. 39, R387 (2006).

14. Y. Cui, C. M. Lieber. Science 291, 851 (2001).

15. H. Yan, H. S. Choe, S. W. Nam, Y. Hu, S. Das, J. F. Klemic, J. C. Ellenbogen, C. M. Lieber. Nature 470, 240 (2011).

16. W. Lu, C. M. Lieber. Nat. Mater. 6, 841 (2007).

17. D. Wang, F. Qian, C. Yang, Z. H. Zhong, C. M. Lieber. Nano Lett. 4, 871 (2004).

18. Y. Cui, Q. Wei, H. Park, C. M. Lieber. Science 293, 1289 (2001).

19. F. Patolsky, G. Zheng, O. Hayden, M. Lakadamyali, X. Zhuang, C. M. Lieber. Proc. Natl. Acad. Sci. USA 101, 14017 (2004).

20. G. F. Zheng, F. Patolsky, Y. Cui, W. U. Wang, C. M. Lieber. Nat. Biotechnol. 23, 1294 (2005).

21. N. S. Ramgir, Y. Yang, M. Zacharias. Small 6, 1705 (2010).

22. S. M. Sze, K. K. Ng. Physics of Semiconductor Devices, $3^{\text {rd }}$ ed., Wiley-Interscience, Hoboken, NJ (2007).

23. M. J. Schoning, A. Poghossian. Analyst 127, 1137 (2002).

24. E. Stern, J. F. Klemic, D. A. Routenberg, P. N. Wyrembak, D. B. Turner-Evans, A. D. Hamilton, D. A. LaVan, T. M. Fahmy, M. A. Reed. Nature 445, 519 (2007).

25. P. Xie, Q. Xiong, Y. Fang, Q. Qing, C. M. Lieber. Nat. Nanotechnol. 7, 119 (2012).

26. M. Arnold, E. A. Cavalcanti-Adam, R. Glass, J. Blummel, W. Eck, M. Kantlehner, H. Kessler, J. P. Spatz. ChemPhysChem 5, 383 (2004).

27. M. Arnold, M. Schwieder, J. Blummel, E. A. Cavalcanti-Adam, M. Lopez-Garcia, H. Kessler, B. Geiger, J. P. Spatz. Soft Matter 5, 72 (2009).

28. J. Park, S. Bauer, A. Pittrof, M. S. Killian, P. Schmuki, K. von der Mark. Small 8, 98 (2012). 
29. J. Park, S. Bauer, K. von der Mark, P. Schmuki. Nano Lett. 7, 1686 (2007).

30. T. Cohen-Karni, K. J. Jeong, J. H. Tsui, G. Reznor, M. Mustata, M. Wanunu, A. Graham, C. Marks, D. C. Bell, R. Langer, D. S. Kohane. Nano Lett. 12, 5403 (2012).

31. N. J. Sniadecki, R. A. Desai, S. A. Ruiz, C. S. Chen. Ann. Biomed. Eng. 34, 59 (2006).

32. C. Prinz, W. Hallstrom, T. Martensson, L. Samuelson, L. Montelius, M. Kanje. Nanotechnology 19, 345101 (2008).

33. G. Cellot, E. Cilia, S. Cipollone, V. Rancic, A. Sucapane, S. Giordani, L. Gambazzi, H. Markram, M. Grandolfo, D. Scaini, F. Gelain, L. Casalis, M. Prato, M. Giugliano, L. Ballerini. Nat. Nanotechnol. 4, 126 (2009).

34. N. A. Kotov, J. O. Winter, I. P. Clements, E. Jan, B. P. Timko, S. Campidelli, S. Pathak, A. Mazzatenta, C. M. Lieber, M. Prato, R. V. Bellamkonda, G. A. Silva, N. W. S. Kam, F. Patolsky, L. Ballerini. Adv. Mater. 21, 3970 (2009).

35. B. Taccardi, B. B. Punske, E. Macchi, R. S. Macleod, P. R. Ershler. Am. J. Physiol. Heart. Circ. Physiol. 294, H1753 (2008).

36. M. L. Cohen, R. H. Hoyt, J. E. Saffitz, P. B. Corr. Am. J. Physiol. 257, H681 (1989).

37. B. P. Timko, T. Cohen-Karni, G. Yu, Q. Qing, B. Tian, C. M. Lieber. Nano Lett. 9, 914 (2009).

38. A. Javey, S. Nam, R. S. Friedman, H. Yan, C. M. Lieber. Nano Lett. 7, 773 (2007).

39. M. C. McAlpine, R. S. Friedman, S. Jin, K. H. Lin, W. U. Wang, C. M. Lieber. Nano Lett. 3, 1531 (2003).

40. T. Takenobu, T. Takahashi, T. Kanbara, K. Tsukagoshi, Y. Aoyagi, Y. Iwasa. Appl. Phys. Lett. 88, 033511 (2006).

41. F. Patolsky, B. P. Timko, G. Yu, Y. Fang, A. B. Greytak, G. Zheng, C. M. Lieber. Science 313, 1100 (2006).

42. T. S. Pui, A. Agarwal, F. Ye, N. Balasubramanian, P. Chen. Small 5, 208 (2009).

43. T. Cohen-Karni, B. P. Timko, L. E. Weiss, C. M. Lieber. Proc. Natl. Acad. Sci. USA 106, 7309 (2009).

44. A. Cohen, J. Shappir, S. Yitzchaik, M. E. Spira. Biosens. Bioelectron. 23, 811 (2008).

45. S. Kodambaka, J. Tersoff, M. C. Reuter, F. M. Ross. Science 316, 729 (2007).

46. C. Y. Wen, M. C. Reuter, J. Bruley, J. Tersoff, S. Kodambaka, E. A. Stach, F. M. Ross. Science 326, 1247 (2009).

47. V. Schmidt, J. V. Wittemann, U. Gösele. Chem. Rev. 110, 361 (2010).

48. C. W. Yang, Z. Zhong, C. M. Lieber. Science 310, 1304 (2005).

49. T. E. Clark, P. Nimmatoori, K.-K. Lew, L. Pan, J. M. Redwing, E. C. Dickey. Nano Lett. 8, 1246 (2008).

50. T. Cohen-Karni, D. Casanova, J. F. Cahoon, Q. Qing, D. Bell, C. M. Lieber. Nano Lett. 12, 2639 (2012).

51. T. Cohen-Karni, Q. Qing, Q. Li, Y. Fang, C. M. Lieber. Nano Lett. 10, 1098 (2010).

52. B. Hille. Ion Channels of Excitable Membranes, $3^{\text {rd }}$ ed., Sinauer (2001).

53. J. M. Dubach, S. Das, A. Rozenzweig, H. A. Clark. Proc. Natl. Acad. Sci. USA 106, 16145 (2009).

54. Q. Qing, S. K. Pal, B. Tian, X. Duan, B. P. Timko, T. Cohen-Karni, V. N. Murthy, C. M. Lieber, Proc. Natl. Acad. Sci. USA 107, 1882 (2010).

55. B. Tian, T. Cohen-Karni, Q. Qing, X. Duan, P. Xie, C. M. Lieber. Science 329, 831 (2010).

56. R. Gao, S. Strehle, B. Tian, T. Cohen-Karni, P. Xie, X. Duan, Q. Qing, C. M. Lieber. Nano Lett. 12, 3329 (2012).

57. X. Duan, R. Gao, P. Xie, T. Cohen-Karni, Q. Qing, H. S. Choe, B. Tian, X. Jiang, C. M. Lieber. Nat. Nanotechnol. 7, 174 (2012).

58. T. C. Pappas, W. M. Wickramanyake, E. Jan, M. Motamedi, M. Brodwick, N. A. Kotov. Nano Lett. 7, 513 (2007). 
59. X. Jiang, B. Tian, J. Xiang, F. Qian, G. Zheng, H. Wang, L. Mai, C. M. Lieber. Proc. Natl. Acad. Sci. USA 108, 12212 (2011).

60. J. T. Robinson, M. Jorgolli, A. K. Shalek, M.-H. Yoon, R. S. Gertner, H. Park. Nat. Nanotechnol. 7, 180 (2012).

61. B. D. Almquist, N. A. Melosh. Proc. Natl. Acad. Sci. USA 107, 5815 (2010).

62. T. Cohen-Karni, R. Langer, D. S. Kohane. ACS Nano 6, 6541 (2012).

63. B. Tian, J. Liu, T. Dvir, L. Jin, J. H. Tsui, Q. Qing, Z. Suo, R. Langer, D. S. Kohane, C. M. Lieber. Nat. Mater. 11, 986 (2012). 